\title{
Optimal Design of Hybrid Optimization Technique for Balancing Inverted Pendulum System
}

\author{
M.A.EBRAHIM ${ }^{1}$, M.E.MOUSA ${ }^{2}$, E.M. SAID ${ }^{1,3}$, M.MAHMOUD ZAKY $^{2}$, S.A.KOTB ${ }^{2}$ \\ ${ }^{1}$ Department of Electrical Engineering, Faculty of Engineering at Shoubra, Benha University, Cairo, EGYPT \\ ${ }^{2}$ Egyptian Second Research Reactor, Egyptian Atomic Energy Authority, Cairo, EGYPT \\ ${ }^{3}$ Head of Electrical Engineering Department, Higher Technological Institute, Cairo, EGYPT
}

\begin{abstract}
Inverted Pendulum system is one of the most exciting problems in control theory. In this research work, a new variant of Grey Wolf optimizer (GWO) via Particle Swarm Optimization (PSO) based on Adaptive Constants (AC) is proposed. The proposed technique (GWO/PSO-AC) is tested via twenty-three benchmark functions and compared to GWO based on PSO without adaptive constants (GWO/PSO). The suggested technique shows superiority in determining the optimal solutions for the well-established benchmark test functions with high computing performance compared to alternative techniques. The proposed GWO/PSO-AC technique, is employed to tune the parameters of the Variable Structure Adaptive Fuzzy (VSAF) controller in addition to the Reduced Linear Quadratic Regulator (RLQR) suggested by the authors. Both controllers are used to stabilize the cart position and to swing up the pendulum angle. The RLQR has an advantage over regular LQR, which is, the numberof the required parameters to obtain the required LQR gains is reduced. The proposed technique is compared with two optimization techniques. The proposed technique achieves high performance for both the cart position and the pendulum angle. The attained results are very promising.
\end{abstract}

Key-Words: Variable Structure Adaptive Fuzzy, Reduced Linear Quadratic Regulator, Inverted Pendulum System, Grey Wolf Optimizer, Particle Swarm Optimization, Harris Hawks Optimizer.

Received: February 16, 2020. Revised: June 8, 2020. Accepted: June 24, 2020. Published: June 30, 2020.

\section{Introduction}

Inverted Pendulum (IP) system is one of the exciting problems in control systems. IP system is used as a test system in various laboratories. It is an unstable, underactuated, non-linear and non-minimum phase system [1]. The concept of the IP system is used in different applications. The idea of the Inverted Pendulum System is used in a lot of new technology applications like aircrafts landing, robots and many other applications in addition to large scale applications such as military applications, transportation and medical applications. Inverted Pendulum (IP) system is also used in industrial applications such as ankle joint control, segways, and quad-rotor helicopter [2]. For nuclear industries, For the IP system, many control techniques are proposed to stabilizing the vehicle and swinging up the pendulum angle [3]. According to the physical components of the system, the balancing situation for the system requires the pendulum to be in a vertical upright position. The position of the pendulum is abnormal. So, permanent force is always required in order to keep the pendulum in a vertically upright position [4]. When the force is stopped, the pendulum will be rotated about the pivot point and be vertically down in an unstable position and the vehicle will move in the opposite direction. So, any change in the position of the pendulum angle will have a direct effect on the position of the vehicle [5]. The main purpose of the proposed controller is to stabilize the vehicle position in addition to swinging up the pendulum angle. The design of controllers must take into consideration that each output of the IP system affects the other one. The proposed controller should be designed and applied to the non-linear system [6].

\section{Related Works}

There are different types of the IP system. Different control techniques are used to balance different types of systems. All types of IP are inherently unstable and non-linear systems. Special control techniques are required to balance these types. The authors in [7] used fuzzy Parallel Distributed Compensation (PDC) to balance the IP system. The authors applied the proposed controller using MATLAB/Simulink. The Linear Matrix Inequality (LMI) is solved to obtain the state feedback gain. Takagi-Sugeno (TS) is used to apply the fuzzy Parallel Distributed Compensation controller. Various disturbances are applied to the system, the controller succeeded in balancing the system under various disturbances. The proposed controller is 
compared to other techniques. The results showed the effectiveness of the proposed controller rather than the other two techniques. Differential equations of the IP system were derived in [8] in order to form the IP model. The input of the system was considered the voltage of the motor. Friction between rail and vehicle was considered. The pendulum succeeded in swinging up in an upright position by using fuzzy swinging up the controller. The system is kept balanced through Sliding Mode Controller (SMC). A comparison between SMC and Linear Quadratic Regulator (LQR) was shown. The results illustrated that SMC is better than using the LQR controller through simulation and experimental results.

The authors in [9] design a controller for balancing the IP system depending on Volterra polynomial basis function networks. The modeling of the IP system was illustrated and discussed. The identification of the IP system through Volterra polynomial basis function networks is achieved using the orthogonal least square algorithm. The special prediction method is introduced in [10] to predict the performance of the IP system. The proposed prediction method was improved rather than traditional ones. The improvement results from a well-learned neural network based on the differences between simulation results and experimental results. The technique is consisting of three main steps. The linear controller for balancing the IP system is designed in the first step. Then, the controller is applied to the system in order to obtain simulation results and experimental results. Through the final step, the differences between both results are used to learn neural networks. The neural network would able to predict the experimental results based on simulation in addition to the difference between them. A comparison between the proposed neural network-basedtechnique and the Linear Quadratic Regulator (LQR) controller is displaced. The results show that the performance of the proposed neural network-basedtechnique is better than LQR.

\section{Mathematical Modeling}

The components of the Cart Inverted Pendulum system are vehicle and arm connected via pivot point. The vehicle moves along the $\mathrm{x}$-axis and the pendulum connected to the vehicle via a pivot point, which rotates freely across the pivot point. The mass center for the system is located above the pivot point. When there is not any force applied to the vehicle, the arm will be rotated about the pivot point until it reaches finally to be vertical down. The proposed control system for the inverted pendulum system shall be able to stabilize the cart position in the desired position and swinging up the pendulum angle in a vertical position. The controller shall ensure robustness for the system. To model the system, the number ofdegree of freedom should be calculated first. For the cart inverted pendulum system, the movement of the cart along the $\mathrm{x}$-axis represents one degree of freedom and swinging up the pendulum is considered the second degree of freedom [11]. Cart moves along $\mathrm{X}$-axis with mass $\mathrm{M}$ by applying the force F. Pendulum is rotating about pivot point with mass $\mathrm{m}$ and has a length equal 21.The parameters of the IP system are displayed in Table 1. The two degree of freedom systems is illustrated inFigure 1. The system differential equations can be represented as follow:

$$
\begin{aligned}
& \ddot{X}=\frac{F_{X}-m g \cos \theta \sin \theta+m l \dot{\theta}^{2} \sin \theta}{M+m \sin ^{2} \theta} \\
& \ddot{\theta}=\frac{(M+m) g \sin \theta-m l \dot{\theta}^{2} \sin \theta \cos \theta-F_{X} \cos \theta}{M l+m l \sin ^{2} \theta}
\end{aligned}
$$

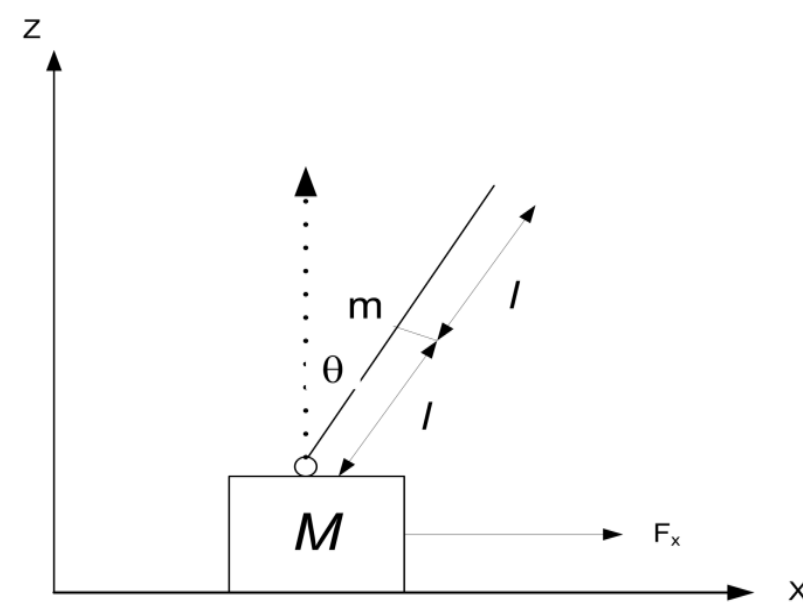

Fig. 1: Modeling of Inverted Pendulum System

Table 1: Parameters of the Cart Inverted Pendulum System

\begin{tabular}{|c|c|c|c|}
\hline Symbol & Parameter & Value & Unit \\
\hline $\mathrm{M}$ & Mass of the cart & 0.455 & $\mathrm{Kg}$ \\
\hline $\mathrm{m}$ & Mass of the pendulum & 0.21 & $\mathrm{Kg}$ \\
\hline $\mathrm{L}$ & $\begin{array}{c}\text { Distance from the pivot to } \\
\text { the mass center of the } \\
\text { pendulum }\end{array}$ & $0.61 / 2$ & $\mathrm{~m}$ \\
\hline $\mathrm{G}$ & Acceleration of gravity & 9.8 & $\mathrm{~m} / \mathrm{s}^{2}$ \\
\hline
\end{tabular}

State representation of the IP system can be obtained according to the values of the system parameters in Table 1. The states of the system are 
described as presented in equation (3). The output states of the system are obtained, as presented by equation (4).

\section{Time-variant state-space representation:}

$$
\begin{aligned}
& \dot{X}(t)=A(t) x(t)+B(t) u(t) \\
& y(t)=C(t) x(t)+D(t) u(t)
\end{aligned}
$$

If the systems are similar in terms of time-invariant, then, we can re-write this equation as the follows:

\section{Time-invariant state-space representation:}

$\dot{X}(t)=A x(t)+B u(t)$

$y(t)=C x(t)+D u(t)$

The state-space representation of the IP system with neglecting the moment of inertia of the pendulum and friction coefficient of the cart is given in equations (7) and (8).

The canonical form of the IP system state space:

$$
\begin{aligned}
& {\left[\begin{array}{l}
\dot{X}_{1} \\
\dot{X}_{2} \\
\dot{X}_{3} \\
\dot{X}_{4}
\end{array}\right]=\left[\begin{array}{cccc}
0 & 1 & 0 & 0 \\
0 & 0 & \frac{-m g}{M} & 0 \\
0 & 0 & 0 & 0 \\
0 & 0 & \frac{(M+m) g}{M l} & 0
\end{array}\right]\left[\begin{array}{l}
X_{1} \\
X_{2} \\
X_{3} \\
X_{4}
\end{array}\right]+\left[\begin{array}{c}
0 \\
\frac{1}{M} \\
0 \\
\frac{-1}{M}
\end{array}\right] F_{X}} \\
& y=\left[\begin{array}{llll}
1 & 0 & 0 & 0 \\
0 & 0 & 1 & 0
\end{array}\right]\left[\begin{array}{l}
X_{1} \\
X_{2} \\
X_{3} \\
X_{4}
\end{array}\right]+\left[\begin{array}{l}
0 \\
0
\end{array}\right] F_{x}
\end{aligned}
$$

After substituting with the values of the parameters of the system, the canonical form state space representation will be as follow:

$\left[\begin{array}{c}\dot{X}_{1} \\ \dot{X}_{2} \\ \dot{X}_{3} \\ \dot{X}_{4}\end{array}\right]=\left[\begin{array}{cccc}0 & 1 & 0 & 0 \\ 0 & 0 & -4.5231 & 0 \\ 0 & 0 & 0 & 0 \\ 0 & 0 & 46.96 & 0\end{array}\right]\left[\begin{array}{l}X_{1} \\ X_{2} \\ X_{3} \\ X_{4}\end{array}\right]+\left[\begin{array}{c}0 \\ 2.198 \\ 0 \\ -7.21\end{array}\right] F_{x}(9)$

$$
\left[\begin{array}{l}
y_{1} \\
y_{2}
\end{array}\right]=\left[\begin{array}{llll}
1 & 0 & 0 & 0 \\
0 & 0 & 1 & 0
\end{array}\right]\left[\begin{array}{l}
X_{1} \\
X_{2} \\
X_{3} \\
X_{4}
\end{array}\right]+\left[\begin{array}{l}
0 \\
0
\end{array}\right] F_{x}
$$

Where:

$$
x_{1}=x, x_{2}=\dot{x}, x_{3}=\theta, x_{4}=\dot{\theta}
$$

\section{Control Techniques}

The advanced control techniques for the inverted pendulum in this research are: variable structure adaptive fuzzy (VSAF), reduced linear quadratic regulator (RLQR) and hybrid GWO/PSO-AC.

\subsection{Variable Structure Adaptive Fuzzy}

The proposed Variable Structure Adaptive Fuzzy (VSAF) controller can tune its' rules with adaptive technique. The controller achieved the defined objective function for the problem based on variable structure methodology. VSAF has a robust estimator because it depends on the variable structure technique. The size of rules is reduced using this technique because it depends on an adaptive rule based on the online results. It does not need to initiate a large number of rules to cover all cases.

On the other hand, the classical Fuzzy Logic Controller (FLC) has three stages to achieve the objective function. The first stage is the fuzzification. In this stage, crisp values are converted then the second stage is Knowledgebase, which contains all the rules. The Defuzzification stage is the final stage of FLC. The main difference between FLC and VSAF is the adaptive rules [12]. FLC has constant rules where the VSAF controller

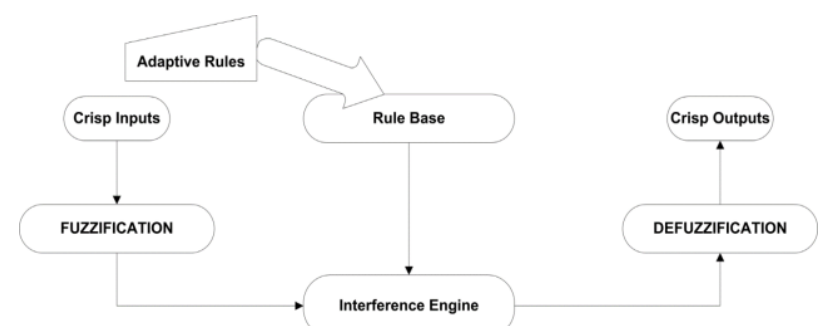

has adaptive rules. The performance of using VSAF is better than using FLC [13]. The structure of the VSAF controller is illustrated in Figure 2.

Fig. 2: Structure of variable structure adaptive fuzzy

\subsection{Reduced Linear Quadratic Regulator:}

Linear Quadratic Regulator (LQR) is based on minimizing a cost function as follow:

$\mathrm{J}=\frac{1}{2} \int_{0}^{\infty}\left[\mathrm{x}^{\mathrm{T}} \mathrm{Qx}+\mathrm{u}^{\mathrm{T}} \mathrm{Ru}\right] \mathrm{dt}$

The above equation has two matrices that be obtained by the designer [14]. After obtaining the two weighting matrices, Riccati equation can be solved according to the following: 


$$
P A+A^{T} P-P B R^{T} B^{T} P+Q=0
$$

The LQR gain can be obtained with the knowledge of weighting matrices in addition to state-space representation matrix of the system as follow:

$\mathrm{K}=\mathrm{R}^{-1} \mathrm{~B}^{\mathrm{T}} \mathrm{P}$

The dynamic modeling of the IP system shows that the order of the system is fourth-order. So, the size of the matrix $\mathrm{Q}$ is $4 * 4$ and the size of matrix $\mathrm{R}$ is $1 * 1$. The number of variables to obtain LQR gain is seventeen parameters. The optimization technique is responsible for finding the appropriate seventeen parameters to obtain the appropriate LQR gain to balance the system.A large number of required parameters to be optimized to obtain LQR gain makes the optimization process complex. In this research work, the new technique is proposed in order to obtain LQR gain, which called RLQR. RLQR is obtained based on less number of parameters than LQR. The size of the Q matrix is equal multiplying $\mathrm{W}$ matrix with size $4 * 2$ by the transpose of $\mathrm{W}$ matrix. Thus, the optimization technique has to optimize eight parameters instead of 16 to obtain the Q matrix. So, the number of parameters reduced from seventeen to nine parameters. The $\mathrm{Q}$ and $\mathrm{R}$ matrices can be obtained as follow:

$$
\begin{aligned}
& \mathrm{Q}=\mathrm{W}^{\mathrm{T}} * \mathrm{~W} \\
& \mathrm{R}=\mathrm{V}^{\mathrm{T}} * \mathrm{~V}
\end{aligned}
$$

Where:

\section{$\mathrm{W}: 4 * 2$ matrix, $\mathrm{V}: 1 * 1$ matrix}

By substitution on the Riccatiequation, it will be as follow:

$$
\mathrm{PA}+\mathrm{A}^{\mathrm{T}} \mathrm{P}-\mathrm{PB}\left(\mathrm{V}^{\mathrm{T}} * \mathrm{~V}\right)^{\mathrm{T}} \mathrm{B}^{\mathrm{T}} \mathrm{P}+\left(\mathrm{W}^{\mathrm{T}} * \mathrm{~W}\right)=0(16)
$$

So, RLQR gain can be obtained through the following:

$\mathrm{K}=\left(\mathrm{V}^{\mathrm{T}} * \mathrm{~V}\right)^{-1} \mathrm{~B}^{\mathrm{T}} \mathrm{P}$

RLQR is obtained through the optimization of seven parameters. RLQR contains four states represented the four states of the system, which are cart position, the velocity of the vehicle, pendulum angle, and angular velocity. RLQR is a feedback gain that is responsible for swinging up the angle. The first parameter is represented by the position of the vehicle. The position of the cart is controlled through VSAF. So, the first parameter of RLQR can be omitted from the reduced gain. The reason for keeping the parameter of vehicle velocity is that the velocity of the vehicle is effect directly on the pendulum angle.

\subsection{Grey Wolf Optimization via Particle Swarm Optimization based on Adaptive Constants (GWO/PSO-AC)}

The Grey wolf with adaptive particle swarm Optimization with an adaptive constant is the proposed optimization technique used to tune the parameters of VSAF and RLQR gain to find the optimal values of the controllers' parameters to balance the IP system.

Grey wolf optimizer is an optimization technique based on social animals that live in packs. Generally, they can be from five to 12 wolves. The wolves are categorized into four levels, which are alpha, beta, delta and omega. Alpha represented the group leaders. They are responsible for taking vital decisions like time of hunting and sleeping. The leaders of wolves not shall be powerful ones, but they are wolves who have high management skills. The supporting wolves are named beta. They are responsible for assisting and supporting the leaders' wolves in their decisions. In case of the absence of alpha wolves due to illness or die, the beta wolves are in the front rows and make the decisions instead of alpha wolves. The delta wolves are responsible for assisting alpha and beta wolves. Delta wolves are elders than other wolves and always protect the pack by the alarm the pack if there is any danger around the pack. Delta wolves have a special methodology in the hunting process by encircling the prey until they stop and then attack the prey. Omega wolves are positioned permanently at the end of the pack. They are the last wolves that can eat among the other wolves. Omega also hasimportant roles as without them, many fights can be occurred among the wolves.

The modeling of grey wolf optimization is discussed in [15]. The mathematical equation of grey wolf optimization technique can be expressed as follow:

$$
\begin{aligned}
& \mathrm{D}_{\alpha}=\left|\mathrm{C}_{1} * \mathrm{X}_{\alpha}(\mathrm{t})\right|, \mathrm{D}_{\beta}=\left|\mathrm{C}_{2} * \mathrm{X}_{\beta}(\mathrm{t})\right|, \\
& \mathrm{D}_{\delta}=\left|\mathrm{C}_{3} * \mathrm{X}_{\delta}(\mathrm{t})\right| \\
& X_{1}=X_{\alpha}-A_{1} * \mathrm{D}_{\alpha}, X_{2}=X_{\beta}-A_{2} * \mathrm{D}_{\beta}, \\
& X_{3}=X_{\delta}-A_{3} * \mathrm{D}_{\delta}
\end{aligned}
$$


$X_{(t+1)}=\frac{X_{1}+X_{2}+X_{3}}{3}$

To obtain optimal values without time-consuming, the GWO technique shall be modified in order to optimal values. GWO is based on random data, which may be led to have poor optimization performance. To enhance the performance of the GWO technique, the velocity of wolves is based on adaptive constants. Grey Wolf Optimization technique via Particle Swarm Optimization based on Adaptive Constant is used to control the IP system. Constants $\mathrm{C}_{1}$ and $\mathrm{C}_{2}$ are effect by the search criteria. The better values are for these constants, the better performance is for the proposed technique. The equations for inertia weight and constants are described as follow:

$$
\begin{aligned}
& \mathrm{w}=\left(\mathrm{w}_{1}-\mathrm{w}_{2}\right) * \frac{\text { Max Iteration-Current Iteration }}{\text { Max Iteration }}+\mathrm{w}_{2}(21) \\
& \mathrm{C}_{1}=2 * \exp \left(-\left(\frac{4 * \text { Current Iteration }}{\text { Max Iteration }}\right)^{2}\right) \\
& \mathrm{C}_{2}=\left(\mathrm{C}_{2 \mathrm{f}}-\mathrm{C}_{2 \mathrm{i}}\right) * \frac{\text { Current Iteration }}{\text { Max Iteration }}+\mathrm{C}_{2}
\end{aligned}
$$

Where:

w: Inertia Weight, w1, w2 represented the lower and higher limits of inertia weight.

$\mathrm{C}_{2 \mathrm{f}}, \mathrm{C}_{2 \mathrm{i}}$ : represents the lower and higher limit of the accelerating coefficient.

Grey wolves have updated their positions via GWO/PSO-AC based on the velocity equation with adaptive constants and inertia weight. The positions of wolves are updated according to the fitness function.

The proposed technique is applied to different functions such as unimodal, multi-model and composite functions. The explanations of functions are illustrated in [16]. Standard deviation and Average are calculated for each 23 functions, as shown inTable 2.According to results, GWO/PSO$\mathrm{AC}$ is better than GWO/PSO. GWO/PSO is better than the proposed technique only for function F12. Figure 3 illustrates the objective functions for all 23 functions for both two techniques.

\subsection{Harris Hawks Optimizer}

Harris Hawks Optimizer (HHO) is an optimization technique inspired by racing in life and called shock jump. Hawks are attacking the prey based on the sudden jump from more than one direction. Themethodology of hawks attacking is based on many factors, such as escaping the process of prey in addition to the attack circumstances. HHO consists of three main stages, which are the exploration phase, the transition from exploration to exploitation and exploitation.

\section{- Exploration Phase}

The criteria of hunting prey using $\mathrm{HHO}$ is introduced in [17] and expressed as follows:

$$
\begin{gathered}
\mathrm{X}_{(\mathrm{t}+1)}= \\
\left\{\begin{array}{c}
\mathrm{X}_{\text {rad }}(\mathrm{t})-\mathrm{r}_{1}\left|\mathrm{X}_{\text {rad }}(\mathrm{t})-2 \mathrm{r}_{2} \mathrm{X}(\mathrm{t})\right| \mathrm{q} \geq 0.5 \\
\left(\mathrm{X}_{\text {rabbit }}(\mathrm{t})-\mathrm{X}_{\mathrm{m}}(\mathrm{t})\right)-\mathrm{r}_{3}\left(\mathrm{LB}+\mathrm{r}_{4}(U B-L B) \mathrm{q}<0.5\right.
\end{array}\right.
\end{gathered}
$$

Where:

$\mathrm{X}_{(\mathrm{t}+1)}$ : Harris Hawks position at iteration $\mathrm{t}+1$.

$\mathrm{X}_{\text {rabbit }}(\mathrm{t})$ : Location of the prey.

$\mathrm{r}_{1}, \mathrm{r}_{2}, \mathrm{r}_{3}, \mathrm{r}_{4}$, and $\mathrm{q}$ : random numbers in the range $(0$, $1)$.

LB: lower limit of the variables, UB: upper limit of the variables.

$\mathrm{X}_{\text {rand }}(\mathrm{t})$ : Hawk selected randomly from the group.

$\mathrm{Xm}$ : average value of the position of the hawks for all the group members.

The average value of the hawks' position can be calculated as follow:

$\mathrm{X}_{\mathrm{m}}(\mathrm{t})=\frac{1}{\mathrm{~N}} \sum_{\mathrm{i}=1}^{\mathrm{N}} \mathrm{X}_{\mathrm{i}}(\mathrm{t})$

$\mathrm{Xi}(\mathrm{t})$ : position of hawks at $\mathrm{t}$ iteration

$\mathrm{N}$ : Total number of hawks.

\section{- The transition from Exploration to Exploitation Phase}

The energy of prey can be described as follow:

$\mathrm{E}=2 \mathrm{E}_{0}\left(1-\frac{\mathrm{t}}{\mathrm{T}}\right)$

Where:

E: Escaping energy of the prey,

$\mathrm{T}$ : Maximum number of iterations,

$\mathrm{E}_{0}$ : the initial state of its energy.

\section{- Exploitation Phase}

The values of $r$ and $E$ generate various cases for this phase. Due to changes in $\mathrm{r}$ and $\mathrm{E}$, four cases exist. For each case, the position of harris hawks is changed due to the change in $\mathrm{E}$ and $\mathrm{r}$ values. 

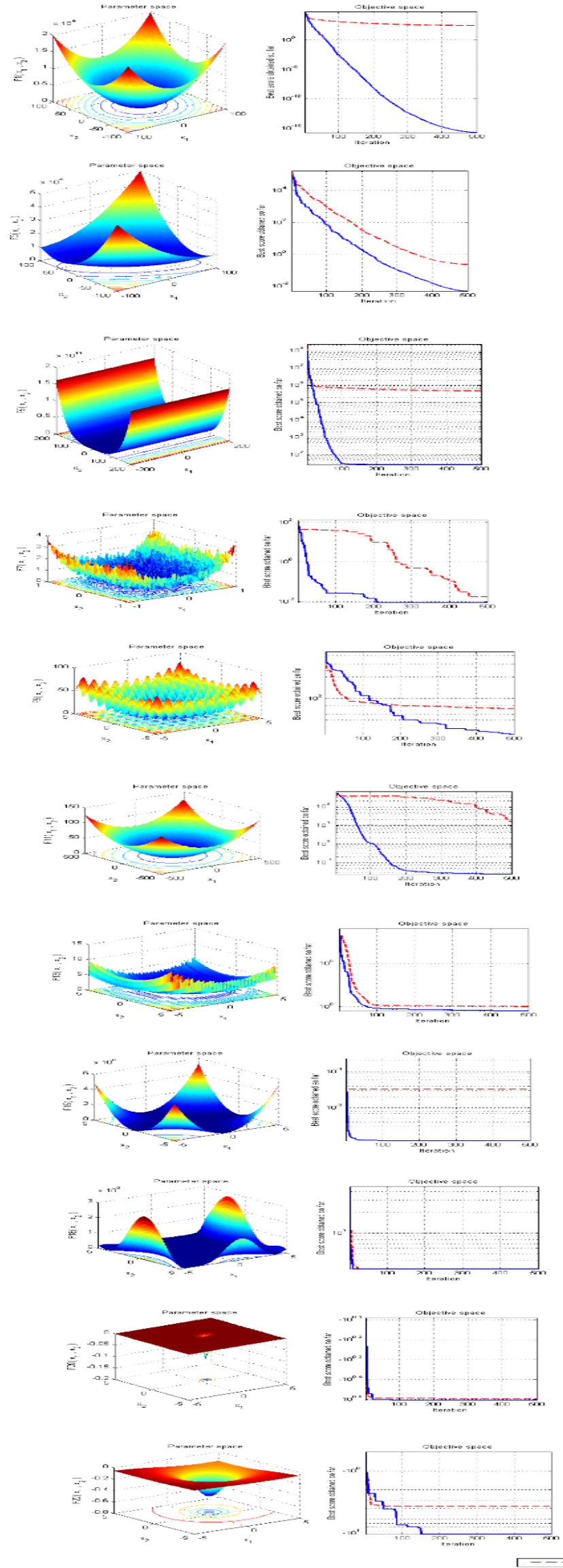

Fig. 3: Convergence curves of GWO/PSO-AC and GWO/PSOtechniques
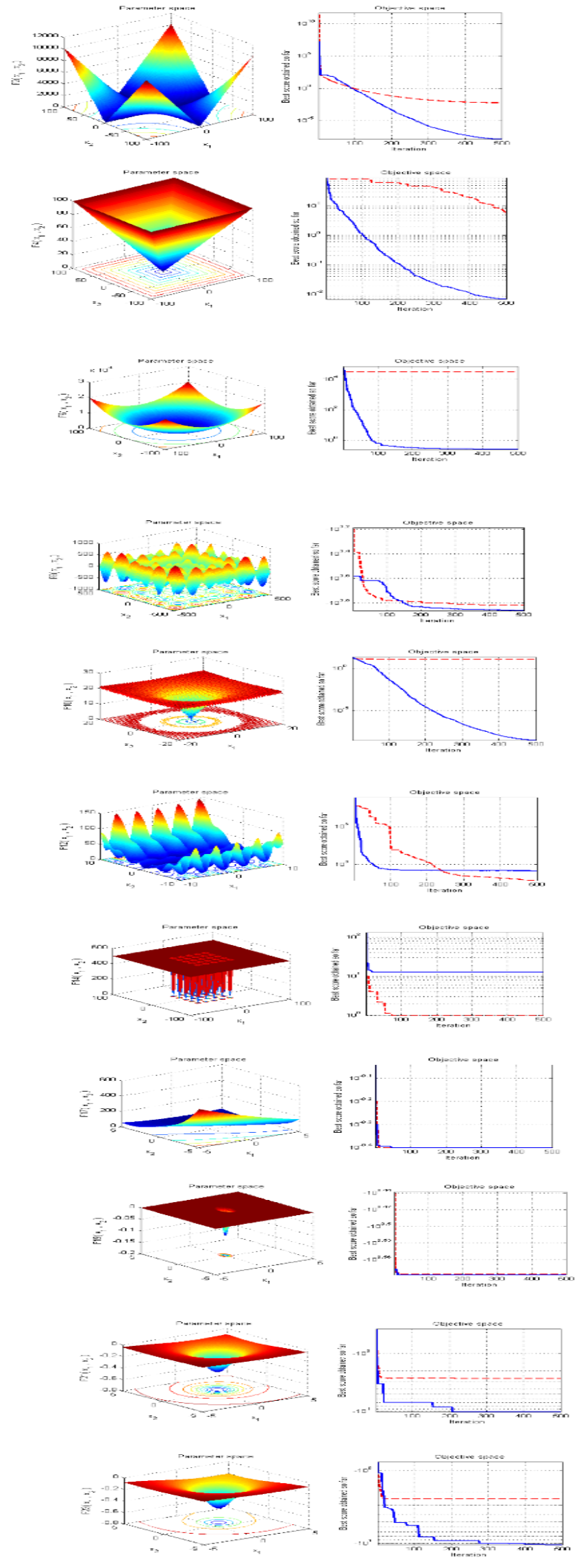
Table 2: Values of Average and Standard Deviation for GWO/PSO and GWO/PSO-AC

\begin{tabular}{|c|c|c|c|c|}
\hline & \multicolumn{2}{|c|}{ GWO/PSO } & \multicolumn{2}{c|}{ GWO/PSO-AC } \\
\hline & Average & Standard Deviation & Average & Standard Deviation \\
\hline F1 & 0.001546 & 0.004074 & $6.30158 \mathrm{E}-16$ & $1.04737 \mathrm{E}-15$ \\
\hline F2 & 0.002565455 & 0.007238576 & $1.69385 \mathrm{E}-08$ & $2.93505 \mathrm{E}-08$ \\
\hline F3 & $5.05 \mathrm{E}-02$ & 0.120548882 & $5.87 \mathrm{E}-03$ & 0.002847178 \\
\hline F4 & 0.072366 & 0.208354 & 0.004844 & 0.004144 \\
\hline F5 & 26.50787 & 0.900079 & 25.96996 & 0.470002 \\
\hline F6 & 0.003161 & 0.005691 & 0.000262868 & $8.77913 \mathrm{E}-05$ \\
\hline F7 & 0.03101 & 0.034465 & 0.007187 & 0.001745 \\
\hline F8 & -6186.49 & 1534.885 & -5519.65 & 2042.702 \\
\hline F9 & 72.57845 & 84.76948 & 21.31398 & 9.48746 \\
\hline F10 & $2.42133 \mathrm{E}-05$ & $5.3996 \mathrm{E}-05$ & $5.65377 \mathrm{E}-09$ & $1.75504 \mathrm{E}-09$ \\
\hline F11 & 0.037802 & 0.031567 & $5.29157 \mathrm{E}-12$ & $1.1293 \mathrm{E}-11$ \\
\hline F12 & 0.001976 & 0.003026 & 0.026263 & 0.033447 \\
\hline F13 & 0.361996 & 0.369308 & 0.132814 & 0.135554 \\
\hline F14 & 1.097428 & 0.314332 & 2.680386 & 1.675966 \\
\hline F15 & 0.006519 & 0.009563 & 0.002782 & 0.006203 \\
\hline F17 & 0.397901763 & $3.25673 \mathrm{E}-05$ & 0.397888587 & $1.77995 \mathrm{E}-06$ \\
\hline F18 & 3.001111 & 0.002427 & 3.000079566 & $6.97922 \mathrm{E}-05$ \\
\hline F19 & -3.85938 & 0.004095 & -3.86198 & 0.002295 \\
\hline F20 & -3.10383 & 0.288695 & -3.29703 & 0.052595 \\
\hline F21 & -7.46469 & 3.575068 & -8.38793 & 2.909308 \\
\hline F22 & -9.52472 & 2.534774 & -9.80941 & 1.770669 \\
\hline F23 & -9.29468 & 2.560123 & -8.41879 & 3.473266 \\
\hline
\end{tabular}

\section{Simulation and Results}

Many various optimization techniques are used to control real-world electromechanical dynamic systems [18-26], especially the IP system [27-29], including computational techniques, and optimal control [30]. IP system is consisting of a vehicle moving along the $\mathrm{x}$-axis. The pendulum is connected to the vehicle through a pivot point. The angle of the pendulum is affected by the movement of the vehicle. The input of the system is the force applied to the vehicle, where the outputs are the cart position and pendulum angle. The VSAF is responsible for stabilizing the cart position where the RLQR is responsible for swinging up the pendulum

angle. The feed-forward gain is responsible for enhancing the performance of the system. Three techniques are proposed in this research work. The first technique is the GWO/PSO. The second technique is the GWO/PSO-AC. The third technique is the HHO. The block diagram of the IP system is shown in Figure 4 and discussed in detail in [31]. Table 3presents the controllers' parameters of each technique. The three techniques are succeeded in stabilizing the cart position and swinging up the pendulum angle. Figure 5 and Figure 6 illustrate the output response of cart position and pendulum angle. 


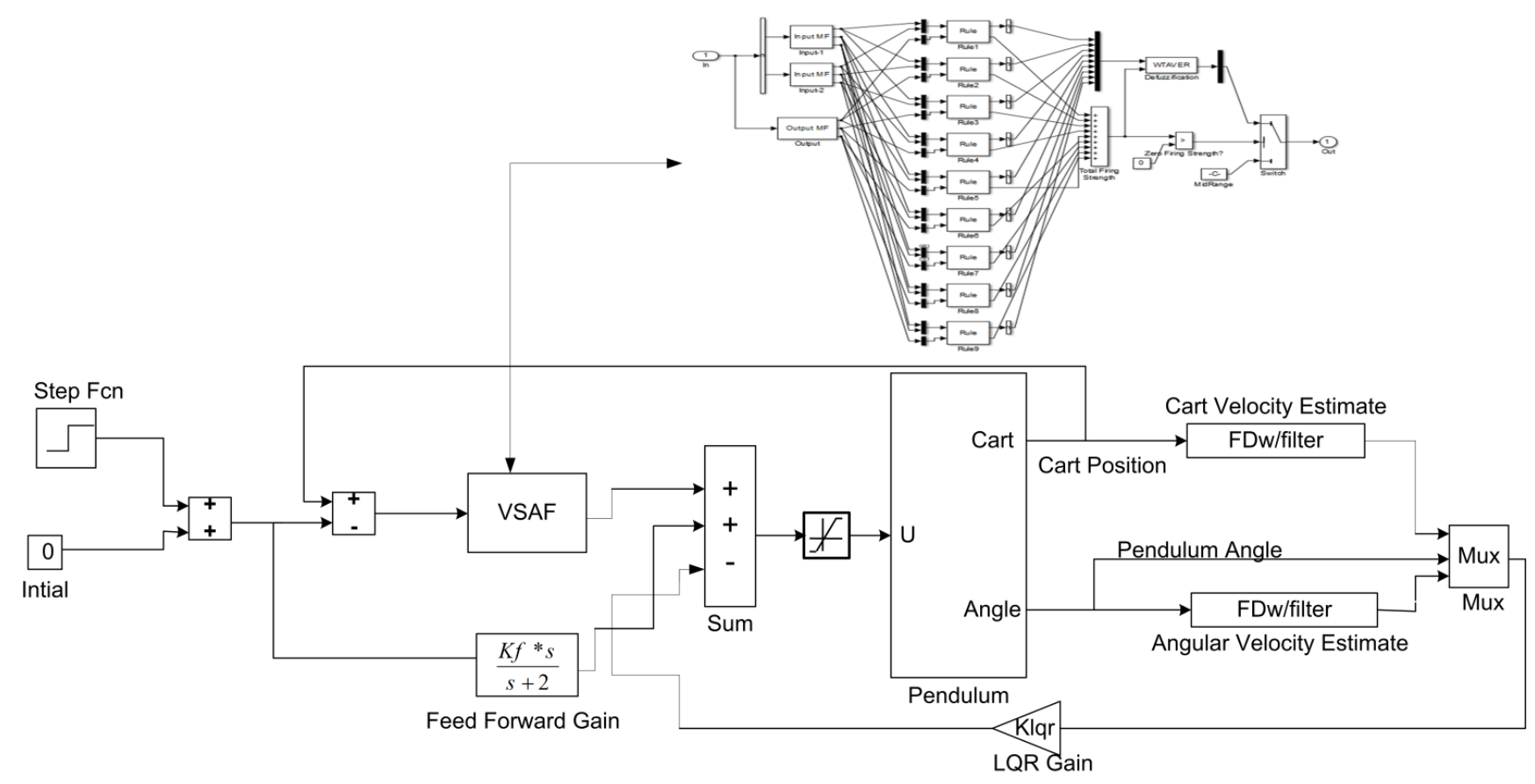

Fig. 4 Block Diagram of an Inverted Pendulum System

Table 3: Gains for Three Techniques

\begin{tabular}{|c|c|c|c|}
\hline & $\begin{array}{c}\mathrm{VSAF} / \\
\mathrm{RLQR} \\
\mathrm{Using} \\
\text { GWO/PSO }\end{array}$ & $\begin{array}{c}\mathrm{VSAF} / \\
\mathrm{RLQR} \\
\text { Using } \\
\text { GWO/PSO-AC }\end{array}$ & $\begin{array}{c}\mathrm{VSAF} / \\
\mathrm{RLQR} \\
\text { Using } \\
\text { HHO }\end{array}$ \\
\hline$K_{b}$ & 32.5 & -33.2 & -0.71 \\
\hline$K_{m}$ & 85.1 & -29.4 & -46.8 \\
\hline$K_{n}$ & 11.4 & 63.5 & 2.91 \\
\hline$K_{f}$ & -13.4 & -3.04 & -2.6 \\
\hline & & {$[-1.5-26.8$} & {$[-1.3-25.9$} \\
$K_{l q r}$ & $\begin{array}{c}{[-6.5-71.1} \\
-19]\end{array}$ & $-12.6]$ & $-11.85]$ \\
\hline
\end{tabular}

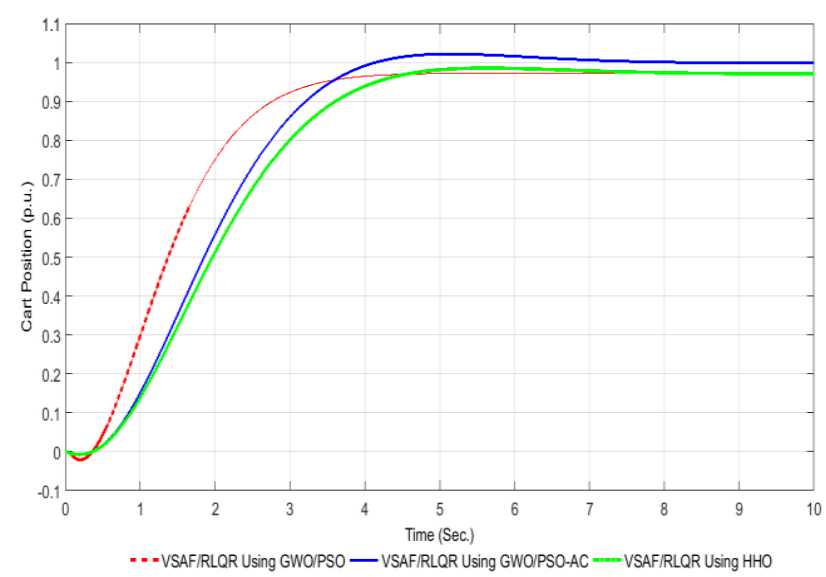

Fig. 5: Cart Position Response Using Three Techniques

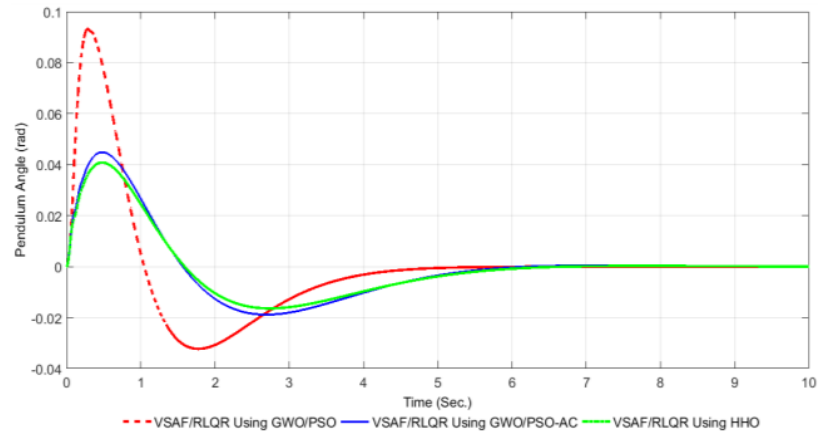

Fig. 6: Pendulum Angle Response Using Three Techniques

The output specifications of the cart position are illustrated inTable 4. GWO/PSO-AC has a better rise time than the other two techniques. Also, GWO/PSO has a minimum settling time amongthe three techniques. The output specifications of the pendulum angle are illustrated inTable 5. The results show that GWO/PSO-AC achieves minimum settling time among the other techniques.

Table 4: Output Specifications for Cart Position

\begin{tabular}{|c|c|c|c|}
\hline O/P & $\begin{array}{c}\text { VSAF/ } \\
\text { RLQR } \\
\text { Using } \\
\text { Specs. }\end{array}$ & $\begin{array}{c}\text { VSAF/ } \\
\text { RLQR } \\
\text { Using } \\
\text { GWO/PSO }\end{array}$ & $\begin{array}{c}\text { VSAF/ } \\
\text { RLQR } \\
\text { Using } \\
\text { HHO }\end{array}$ \\
\hline Rise time & 2 & 2.4 & 2.6 \\
\hline Sett. time & 3.7 & 5.8 & 4.3 \\
\hline Ov. sht & 0 & 2.4 & 1.2 \\
\hline Un. sht & 2.1 & 0.76 & 0.69 \\
\hline
\end{tabular}


Table 5: Output Specifications for Pendulum Angle

\begin{tabular}{|c|c|c|c|}
\hline $\begin{array}{c}\text { Time } \\
\text { response }\end{array}$ & $\begin{array}{c}V S A F / R L Q R \\
\text { Using } \\
\text { GWO/PSO }\end{array}$ & $\begin{array}{c}V S A F / R L Q R \\
\text { Using } \\
\text { GWO/PSO- } \\
\text { AC }\end{array}$ & $\begin{array}{c}\text { VSAF/ } \\
\text { RLQR } \\
\text { Using } \\
\text { HHO }\end{array}$ \\
\hline $\begin{array}{c}\text { Maximum } \\
\text { value }\end{array}$ & 0.0942 & 0.045 & 0.04 \\
\hline $\begin{array}{c}\text { Minimum } \\
\text { value }\end{array}$ & -0.0323 & -0.019 & -0.016 \\
\hline $\begin{array}{c}\text { Settling } \\
\text { time }\end{array}$ & 4.36 & 5.7 & 6.2 \\
\hline
\end{tabular}

To check the robustness of the controllers with different techniques, some tests can be applied to the IP system to ensure the effectiveness of the proposed controllers. The first test is increasing the step input applied to the system. The results are shown in Figure 7 and Figure 8. The results show that only GWO/PSO-AC and VSAF/RLQR using $\mathrm{HHO}$ are succeeded in balancing the system.

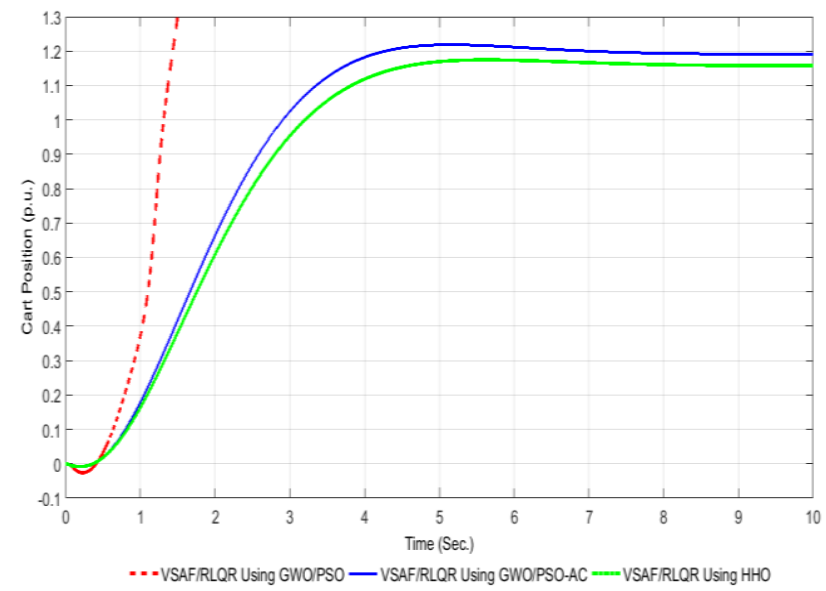

Fig. 7: Cart Position Response with 120\% Step Input

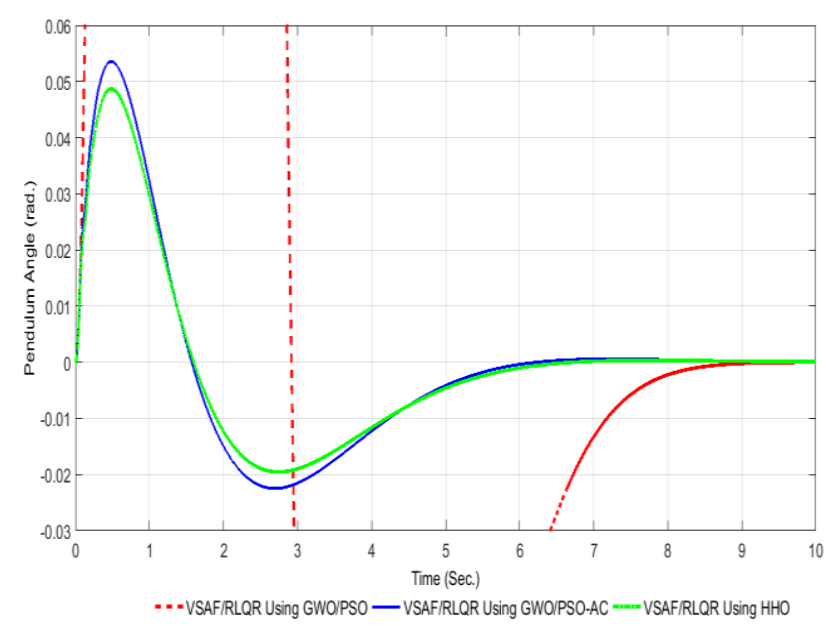

Fig. 8: Pendulum Angle Response with 120\% Step Input
The second test is related to the parameters of the system. Due to uncertainties, the mass of pendulum may be changed in case of a cup of glass put on the pendulum. The results are shown in Figure 9 and Figure 10 . The results show that only VSAF/RLQR using GWO/PSO-AC and VSAF/RLQR using HHO are succeeded in balancing the IP system.

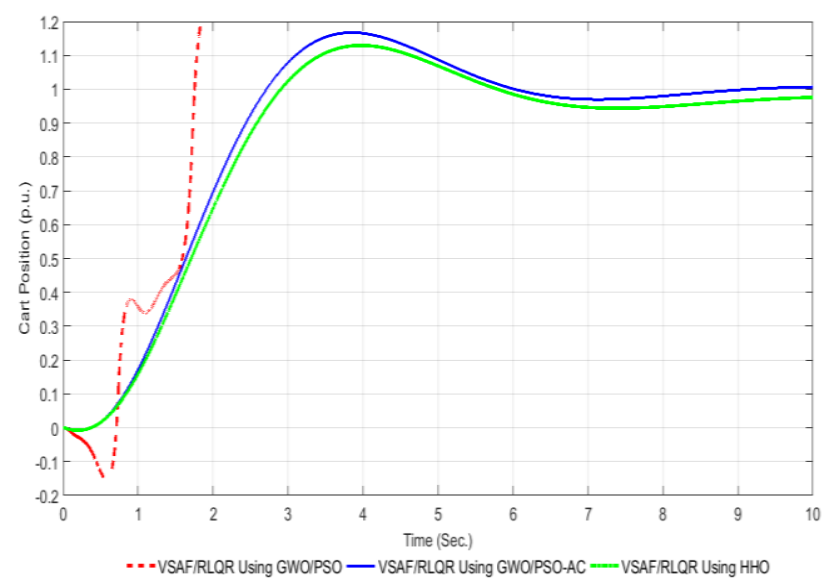

Fig. 9: Cart Position Response with $1 \mathrm{Kg}$ Mass Pendulum

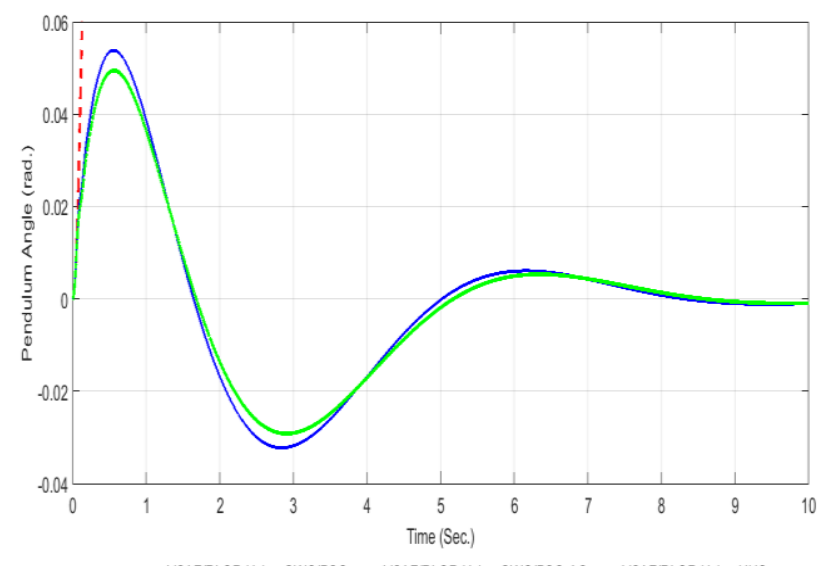

- -VSAFRLQR Using GWOPSO —VSAFRLQR Using GWOPSO-AC =---VSAFIRLQR Using HHO

Fig. 10: Pendulum Angle Response with 1Kg Mass Pendulum

\section{Conclusions}

In this research work, a new variant of Grey Wolf optimizer (GWO) via Particle Swarm Optimization (PSO) based on Adaptive Constants (AC) is developed and tested using twenty-three benchmark functions to check its validity before applying to the inverted pendulum system. Moreover, Variable Structure Adaptive Fuzzy (VSAF) with Reduced Linear Quadratic Regulator (RLQR) is suggested to control the Inverted Pendulum (IP) system. Both controllers, in addition, to feed-forward gain,are tuned through the proposed technique of GWO/PSO-AC. The proposed technique succeeded in balancing the system effectively with robustness 
tests. The first robustness test is increasing the step input by $120 \%$, where the second robustness test is changing the pendulum mass value to be $1 \mathrm{Kg}$. The proposed technique is compared with two other optimization techniques. The results are promising for real-time applications. As a prospective for this article, the new variant of GWO paves the way for real-time implementation due to its high computing performance in solving different optimization problems. Besides, the proposed VSAF/RLQR control topology can be utilized to control different electromechanical systems.

\section{References}

[1]Mondal R, Chakraborty A, Dey J, Halder S. Optimal fractional-order PI $\lambda \mathrm{D} \mu$ controller for stabilization of cartinverted pendulum system: Experimental results. Asian Journal of Control. 2019.

[2]Blondin MJ, Pardalos PM. A holistic optimization approach for inverted cartpendulum control tuning. Soft Computing. 2020 Mar;24(6):4343-59.

[3] Hanwate S, Hote YV, Budhraja A. Design and implementation of adaptive control logic for cartinverted pendulum system. Proceedings of the Institution of Mechanical Engineers, Part I: Journal of Systems and Control Engineering. 2019 Feb;233(2):164-78.

[4] Ibanez CA, Garcia JC, Lopez AS, de Jesus Rubio J, Castanon MS. Stabilization of the inverted cart-pendulum system with linear friction. IEEE Latin America Transactions. 2018 Aug 22;16(6):1650-7.

[5] Issa A, El Shorafa M, Aqel MO, Brabazon D, Young P. Remote Computer-Based Learning System for Inverted Pendulum Lab Experiment. In2018 International Conference on Promising Electronic Technologies (ICPET) 2018 Oct 3 (pp. 113-117). IEEE.

[6]Mousa ME, Ebrahim MA, Hassan MM. Stabilizing and swinging-up the inverted pendulum using PI and PID controllers based on reduced linear quadratic regulator tuned by PSO. International Journal of System Dynamics Applications (IJSDA). 2015 Oct 1;4(4):52-69.

[7]Roose AI, Yahya S, Al-Rizzo H. Fuzzy-logic control of an inverted pendulum on a cart. Computers \& Electrical Engineering. 2017 Jul $1 ; 61: 31-47$.

[8]Elsayed BA, Hassan MA, Mekhilef S. Fuzzy swinging-up with sliding mode control for thirdorder cart-inverted pendulum system.
International Journal of Control, Automation and Systems. 2015 Feb 1;13(1):238-48.

[9] Ronquillo-Lomeli G, Ríos-Moreno GJ, GómezEspinosa A, Morales-Hernández LA, Trejo-Perea M. Nonlinear identification of inverted pendulum system using Volterra polynomials. Mechanics Based Design of Structures and Machines. 2016 Apr 2;44(1-2):5-15.

[10] Khalid J, Nasir A, Shami U, Baig A. Using Denoising Autoencoders to Predict Behavior of an Inverted Pendulum on a Cart System. University of Engineering and Technology Taxila. Technical Journal. 2017;22(1):30.

[11] Strakoš P, Tůma J. Mathematical modelling and controller design of inverted pendulum. In2017 18th International Carpathian Control Conference (ICCC) 2017 May 28 (pp. 388-393). IEEE.

[12] Hua C, Guan X, Duan G. Variable structure adaptive fuzzy control for a class of nonlinear time-delay systems. InProceedings of the 2004 American Control Conference 2004 Jun 30 (Vol. 1, pp. 476-481). IEEE.

[13] Ebrahim, M.A. and Ramadan, H.S.Interarea power system oscillations damping via AI-based referential integrity variable-structure control. International Journal of Emerging Electric Power Systems, 2016, 17(5), pp.497509.

[14] Levine WS. Linear quadratic regulator control. InThe Control Systems Handbook 2018 Oct 3 (pp. 403-426). CRC Press.

[15] Saremi S, Mirjalili SZ, Mirjalili SM. Evolutionary population dynamics and grey wolf optimizer. Neural Computing and Applications. 2015 Jul 1;26(5):1257-63.

[16] Mirjalili S, Mirjalili SM, Lewis A. Grey wolf optimizer. Advances in engineering software. 2014 Mar1;69:46-61.

[17] Heidari AA, Mirjalili S, Faris H, Aljarah I, Mafarja M, Chen H. Harris hawks optimization: Algorithm and applications. Future generation computer systems. 2019 Aug 1;97:849-72.

[18] Ebrahim, M.A., Ramadan, H.S. and Soliman, M.Robust non- fragile approach to resilient design of PID- based blade pitch control for wind energy conversion system. Asian Journal of Control,2019, 21(4), pp.1952-1965.

[19] Aouchiche, N., Aitcheikh, M.S., Becherif, M. and Ebrahim, M.A.AI-based global MPPT for partial shaded grid connected PV plant via MFO approach. Solar Energy, 2018, 171, pp.593-603.

[20] Ebrahim, M.A. and Mohamed, R.G.Comparative study and simulation of different maximum power point tracking 
(MPPT) techniques using fractional control \& grey wolf optimizer for grid connected pv system with battery. In Electric Power Conversion. IntechOpen, 2019.

[21] Ebrahim, M.A., Osama, A., Kotb, K.M. and Bendary, F.Whale inspired algorithm based MPPT controllers for grid-connected solar photovoltaic system. Energy Procedia, 2019, 162, pp.77-86.

[22] Helal, S.A., EBRAHIM, M., RADY, N.M. and SALAMA, M.M.WHALE OPTIMIZATION ALGORITHM BASED OPTIMAL MPPT OF PV POWER PLANT (REAL CASE STUDY). Journal on Electrical Engineering, 2019, 12(3).

[23] Ebrahim, M.A., Becherif, M. and Abdelaziz, A.Y.Dynamic performance enhancement for wind energy conversion system using MothFlame Optimization-based blade pitch controller. Sustainable Energy Technologies and Assessments, 2018, 27, pp.206-212.

[24] Ebrahim, M.A., Elyan, T., Wadie, F. and AbdAllah, M.A.Optimal design of RC snubber circuit for mitigating transient overvoltage on VCB via hybrid FFT/Wavelet Genetic approach. Electric Power Systems Research, 2017, 143, pp.451-461.

[25] Maher, M., Ebrahim, M.A., Mohamed, E.A. and Mohamed, A.Ant-lion optimizer based optimal allocation of distributed generators in radial distribution networks. International Journal of Engineering and Information Systems, 2017, 1(7), pp.225-238.
[26] Omar, M., Ebrahim, M.A., Ghany, A.A. and Bendary, F.Tuning of PID controller for load frequency control problem via harmony search algorithm. Indonesian Journal of Electrical Engineering and Computer Science, 2016, 1(2), pp.255-263.

[27] Hassanzadeh I, Mobayen S. Controller design for rotary inverted pendulum system using evolutionary algorithms. Mathematical Problems in Engineering. 2011;2011.

[28] Mladenov V. Application of neural networks for control of inverted pendulum. WSEAS Transactions on Circuits and Systems. 2011 Feb 1;10(2):49-58.

[29] Duka AV, Oltean SE, Dulau M. Model reference adaptive control and fuzzy model reference learning control for the inverted pendulum. Comparative analysis. InProceedings of WSEAS International Conference on Dynamical Systems and Control 2005 Nov 2 (pp. 168-173).

[30] Prasad LB, Tyagi B, Gupta HO. Optimal control of nonlinear inverted pendulum system using PID controller and LQR: performance analysis without and with disturbance input. International Journal of Automation and Computing. 2014 Dec 1;11(6):661-70.

[31] Ciprian, P. P., Luminita, D., \& Lucia, P. (2011). Control optimization using MATLAB. In Engineering Education and Research Using MATLAB. IntechOpen.

\section{Creative Commons Attribution \\ License 4.0 (Attribution 4.0 \\ International , CC BY 4.0)}

This article is published under the terms of the Creative Commons Attribution License 4.0 https://creativecommons.org/licenses/by/4.0/deed.en US 